\title{
The application of Function Analysis in development of rehabilitation product
}

\author{
Changqing Gao ${ }^{a,{ }^{*}}$, Wei Wang ${ }^{a}$, Jianhua Sun ${ }^{a}$ and Peng Chao ${ }^{b}$ \\ a School of Mechanical Engineering, University of Jinan, No.336 Nanxinzhuang West Road, Jinan, \\ Shandong, P.R.China \\ ${ }^{\text {b }}$ Haier Medical and Laboratory Products Co., Ltd, No.1 Haier Road, Qingdao, Shandong, \\ P.R.China
}

Keywords: rehabilitation engineering, function analysis, new product development

\begin{abstract}
Rehabilitation product is something that can help the persons with disabilities to solve the challenges with necessary functions. The development of rehabilitation product is the important subject with complex process in rehabilitation engineering, which would use cross field knowledge. Conceptual design is the key process for new product and function analysis is the main job for the engineers to locate the solutions. The result of conception design is to generate design scheme. The function analysis usually contents function definition, function decomposition, function chain analysis and function model. Finally, the process and significance of function analysis is illustrated with an example rehabilitation robot. Function analysis can help the rehabilitation engineers for problem analyzing and it is the base for the detailed design in the next step.
\end{abstract}

\section{Introduction}

New product development is important for the indigenous innovation ability of enterprises. The essence of design is innovation. Rehabilitation product can meet the living requirements and improve the life quality of the persons with disabilities. So it has caused the widespread concern of social public [1]. To develop the rehabilitation product is one of the important tasks for the rehabilitation engineers, which usually use the different domain knowledge. The application of design methodology in the rehabilitation product development is a necessary tool to promise the design quality.

The result of conception design is generating design scheme and the formation of effective Principe solutions is an important output form of concept stage. So scheme design is an important content in the process of conception design. This process is closely related to function, behavior and the mapping between structures, it is a reasoning process based on knowledge [2].

Design theory and method is discipline which studies essential rules, step and strategy of product design. Quality Function Deployment (QFD), Axiomatic Design (AD) and Systematic Approach of Pahl and Beitz (SAPB) are all research results of this field. SAPB was a representative of the German classical design theory. It comprehensively analyzes the design process, and puts forward a complete design methodology. There is great practical value to guide product design. The function analysis of SAPB and its application in the rehabilitation product design are discussed in this paper.

\section{Systematic Approach of Pahl and Beitz (SAPB)}

\subsection{Scheme design and conception design}

SAPB divided the design flow into four stages: clarify the task, scheme design, technical design and construction design. Although there is significant difference on knowing about the research categories and implementation of conception design in domestic scholars, most of them agrees that scheme design is the main content of conceptual design. According to the requirements, we can get corresponding design scheme by various methods [3]. So researching the method to obtain design scheme is an important content to realize the conceptual design. 


\subsection{The process of SAPB scheme design}

The process of scheme design generally includes the following contents: using black box method to abstract design task and determining the total function of a system based on the analysis of demand table, according to the change of material flow, energy flow and signal flow to determine the logical relationship and spatial relationship among the various points function and then form the function structure, looking for physical effects and its geometry, material characteristics that could meet the demand of various points function, then form the action principle, forming technology solution on the basis of compatibility principle based on the morphology matrix, evaluating and screening scheme under the condition of design constraints [4].

The acquisition of action principle is the basis to form design scheme. Looking for the principle to realize the various points function is a process to generally refine design scheme. Action principle provides preliminary structural features for the subsequent design work and it is closely related to the application of science effect and empirical knowledge in the field, as shown in Fig. 1.

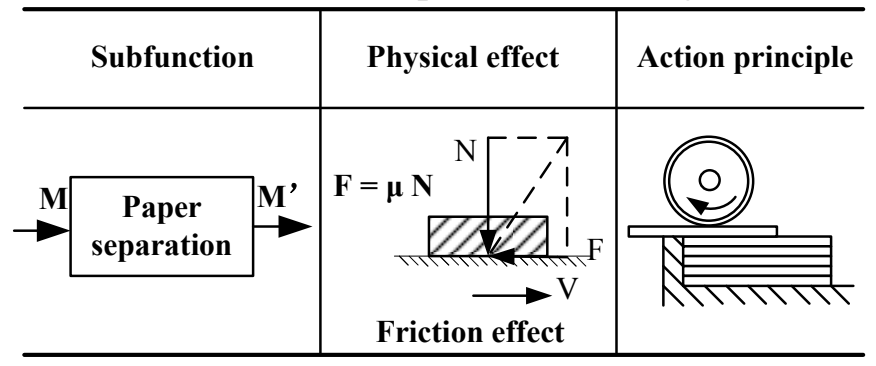

Fig. 1. Function, physical effect and action principle.

\section{Function analysis}

The core of product function design is according to the function requirements to realize the scheme solution of specific function at the level of principles, including the conception of new function, function analysis, function structure design, new principle innovation of function, structure innovation of function unit, structure solution combination, etc.

\subsection{The function definition}

The function abstractly describes the causality of mechanical products between input and output. For the specific products, function refers to the efficiency, purpose and effect of product. What we purchased is product function and what we used is also product function. For example, the function of light is to convert electrical energy into light energy and the function of lathe is to realize metal cutting. The function of induction cooker is to convert electrical energy into heat energy, etc. When using function analysis to make scheme design, we generally according to the following steps: (1) abstract design task, determine a total function, seize the essence, extended thinking and look for many ways to solve problems, (2) divide the total function into function unit, and form function tree, (3) seeking to function (function unit) solution, (4) scheme evolution and decision [5].

From the perspective of system theory, we define the function as the relationship of technology system between input and output. For the problem to be solved, it is difficult to know immediately for the designers, and just like treat an opaque "black box" which of inner structure is uncertain. Using external observation to unknown system, we can analyze the input and output between this system and environment, and then determine the functional feature of this system, thus seek the function solution. This method is called "black box". "Black box" requires designer to design the products from the system function rather than the product structure. This is a transformation of aggregate method. "Black box" is advantageous to grasp the essence of problem, expand the thinking, get rid of the traditional structure and get an innovative and high-level design scheme. Generally speaking, the input and output of technology system divided into three forms: material, signals and energy, as shown in Fig. 2. 


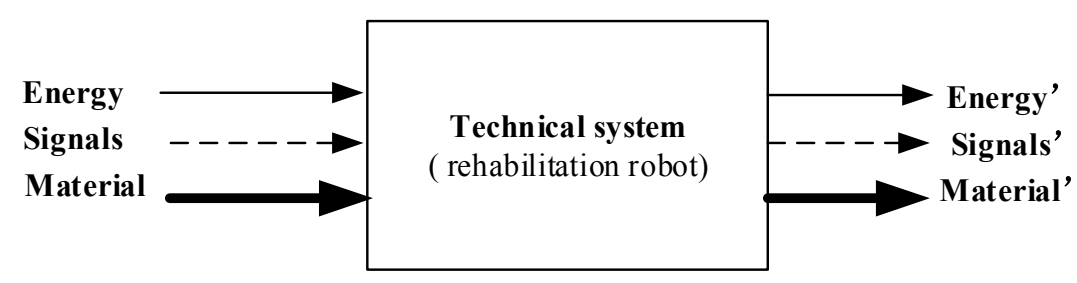

Fig. 2. The expression of function.

\subsection{Functional decomposition}

Function unit is the basic unit of the function solving process. The most common basis units include physical function unit, logic function unit and mathematical function unit.

Physical function unit. It reflects the physical basic action of energy, material and signal in the system, more common are: shift-recovery, zoom-narrow, connections-separation, conductioninsulation, storage-extraction.

Mathematical function unit. It reflects the fundamental action of mathematics, such as addition, subtraction, multiplication, division, involution, evolution, differential and integral. The mathematical function unit is mainly used on mechanical addition and subtraction structure and evolution structure, such as differential gear train, computer, etc.

Logic function unit. It includes "and", "or", "not" ternary logic action, and it mainly used to control function [6].

\subsection{Function modeling}

The establishment of functional model is the original and also the important step in the process of scheme design. It determines the total function by analyzing the user requirements and divides the total function into various points function until function unit. Function unit is realized by known parts, process or subsystem. Functional model is the most direct expression of product design knowledge and design intent and it plays an important role in product analysis and design. Functional model is one of the important links to make system from abstract to concrete [7].

Through the establishment of functional model, we defined the various points function, function unit and their sequence that realized the total function of system. In the next scheme design, these simple function or function unit can easily solve each function unit through the function structure mapping, thus getting the solid solution that needed to realize the total function.

The steps of function model are illustrated as below.

Determine the total function of system. The total function is when energy flow, material flow and signals flow expressed in block diagrams through the technology system. Designers start out from design requirements through the analysis and summary of design requirements and build the requirement model of product. Then we start out from the requirement model, using "black box" to abstract total function of this product.

Decompose the total function into sub function step-by-step and establish function tree.

Establish function train according to input flow and output flow. Considering each operation from input to output or conversion and according to the conversion sequence to range each function units, then establish function train according to input stream and output stream.

Build functional model based on the above function tree and function train.

In the actual design process, we start out from the requirement model of design to abstract the total function of system. How to decompose the total function of system and decompose to what degree is a complex problem. This process depends on many factors, such as the specific conditions and complexity of the problem, the experience and purpose of designers, etc.

\section{Case study}

Persons with serious disabilities face much challenge in their life. They need some rehabilitation trainings to help them for the recovery of muscles, bones, joints, etc. If patients are in serious health conditions, the caretaker is usually needed for disease treatment to help them to finish some actions. Rehabilitation robot is a device for rehabilitation trainings and it is a good helper for the caretakers. 
Taking the design of rehabilitation robot as an example, we described the steps of function analysis and functional model establishment in the rehabilitation product design.

(1) Determining the total function of the system. Determining the input and output flow and abstracting the total function of products applied with "black box". As shown in Fig. 2.

(2) Decomposing the total function of products step by step and building the functional decomposition tree, as shown in Fig. 3.

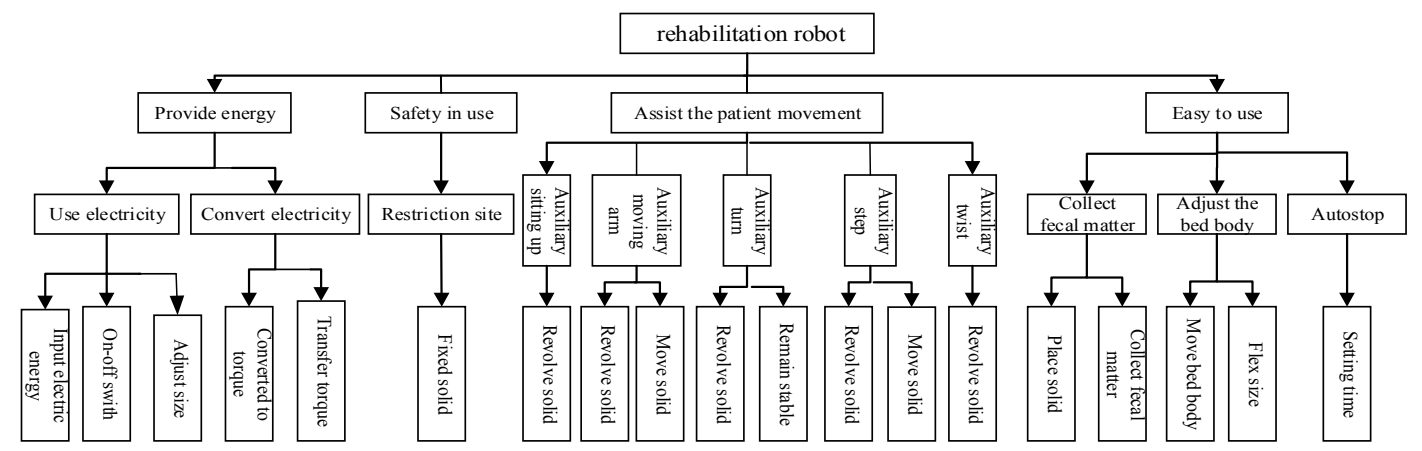

Fig. 3. The functional decomposition tree.

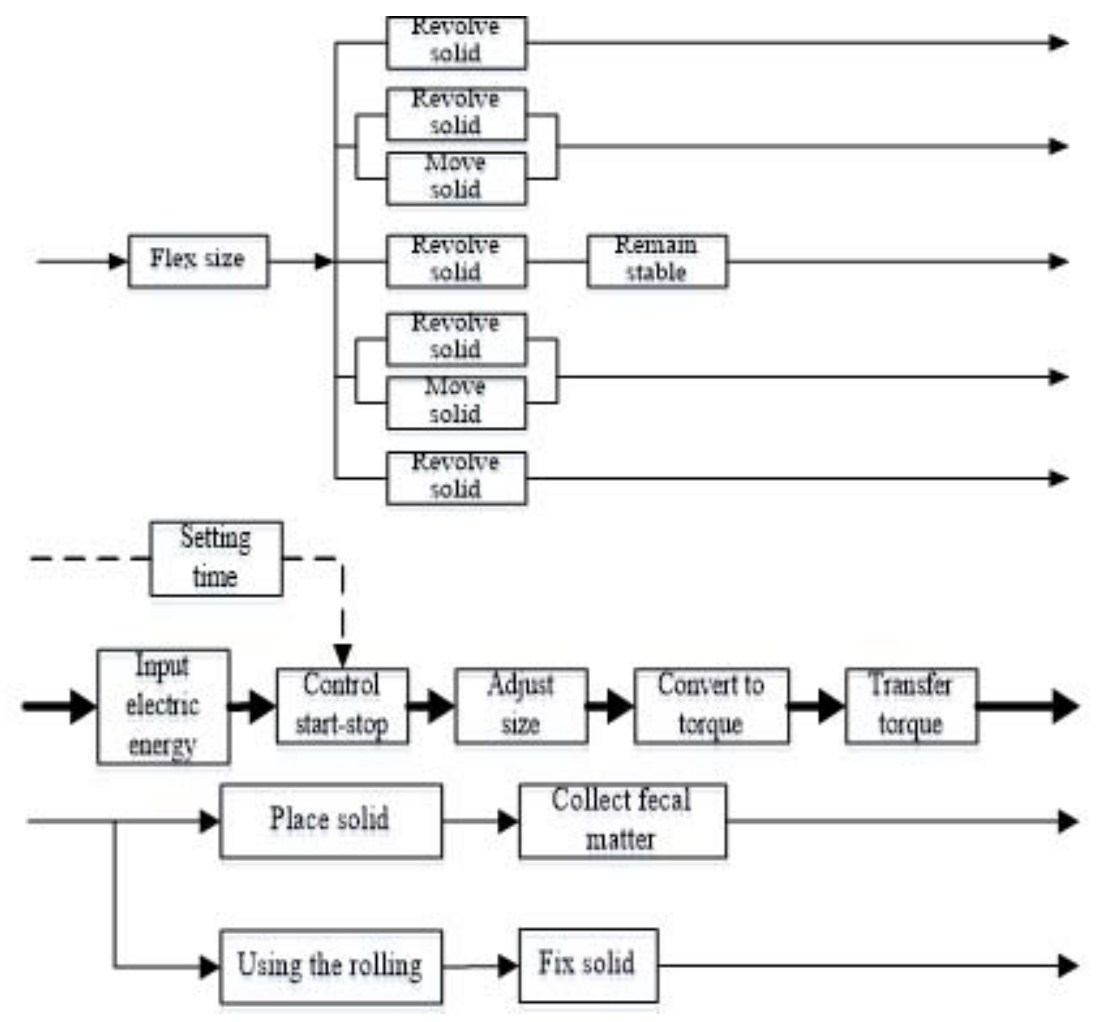

Fig. 4. The function chain.

(3) Establishing a function chain for each input flow. Considering each operation from input to output or conversion, according to the chronological order of the operation, we ranged every function unit and established function chain, as shown in Fig. 4. 


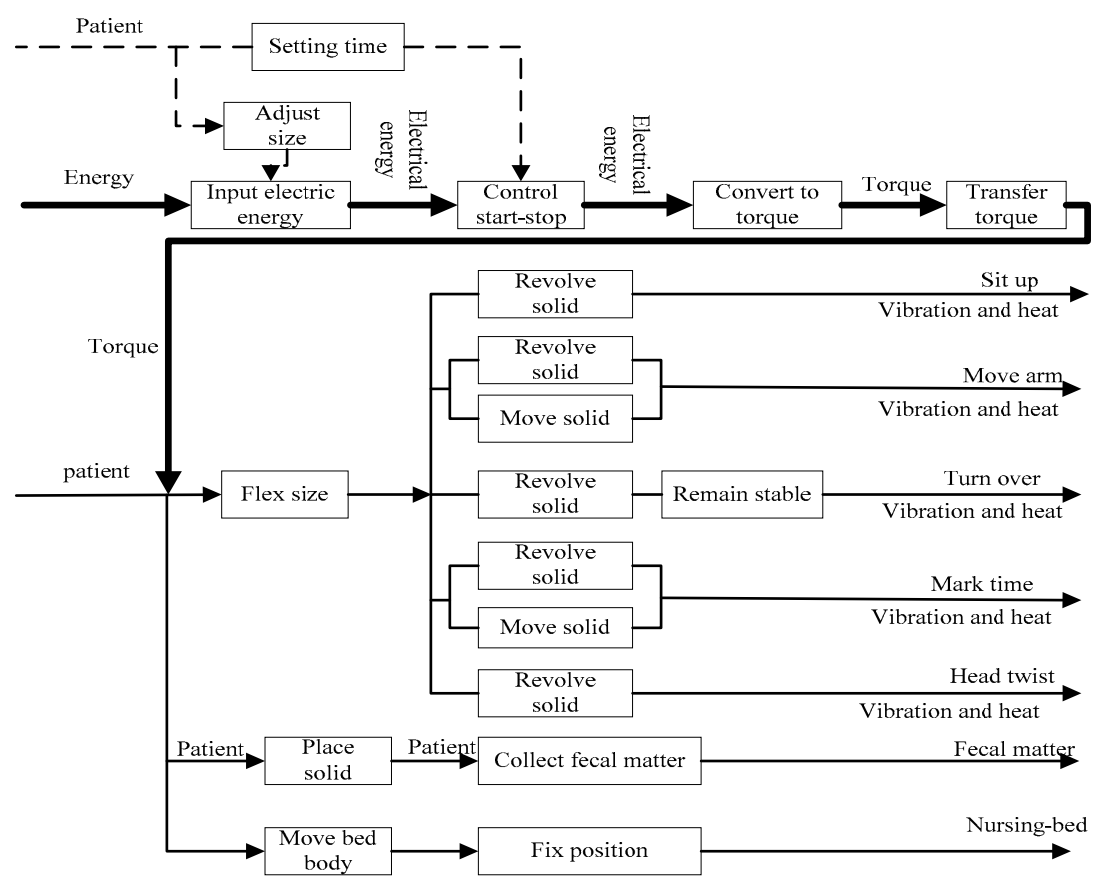

Fig. 5. The functional model.

(4) Connecting the function chain and getting the functional model, as shown in Fig. 5. The 3D model of rehabilitation robot is shown in Fig. 6.

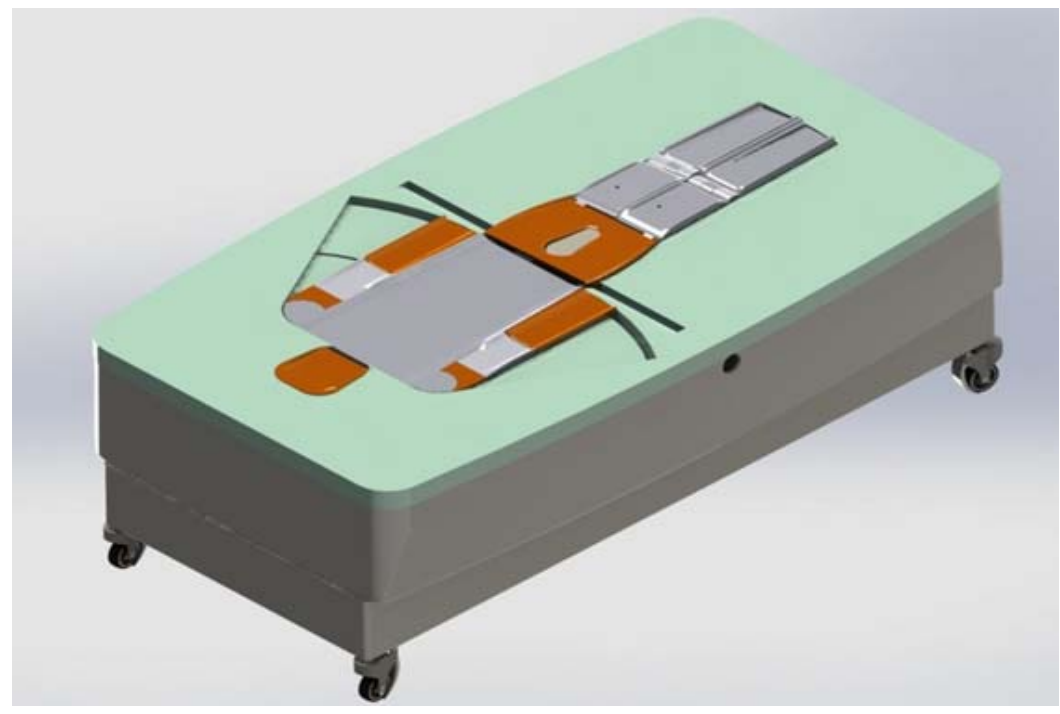

Fig. 6. 3D model of rehabilitation robot.

\section{Conclusions}

(1) Function is the nature of the product and the basis to realize the product innovation. Function analysis is one of the key steps in the process of product conception design. Most design theory and methods make the function analysis as an important part of the model design process. Functional decomposition, function unit solution and the determination of function structure are the main contents of function analysis.

(2) Rehabilitation product development is a process for inventive problem solving with cross domain knowledge. The applications of Function Analysis can impulse the innovation design of the product. 


\section{Acknowledgements}

This work was financially supported by Science and Technology Development Plan Project of Shandong Province (2014GGX106003), Large Equipment Upgrade Project of Shandong Province (2012SJGZ15).

\section{References}

[1] Miao Li , Xinguo Ming, Lina He, Maokuan Zheng, Zhitao Xu, A TRIZ-based Trimming method for Patent design around, Computer-Aided Design, 62 (2015), 20-30.

[2] Runhua Tan, A Sequential Process Model for Idea Generation in Fuzzy Front End using TRIZ, Journal of TRIZ in Engineering Design, 2(1), (2006), 59-73.

[3] PAHL G, BEITZ W. Engineering design: a systematic approach, London: Springer-Verlag, 2007.

[4] L. TANG, The fundamental methodology for conceptual design, Beijing: National Defence Industry Press, 2006.

[5] CH. Q. GAO. TRIZ - Theory of inventive problem solving, Beijing: Science Press, 2011.

[6] LIANG Yanhong, TAN Runhua. Functional Analysis in Conceptual Design of Products, Journal of Hebei University of Technology, 36(4), (2007), 33-38. 